DOI: 10.1002/adfm.((please insert DOI)

Submitted to

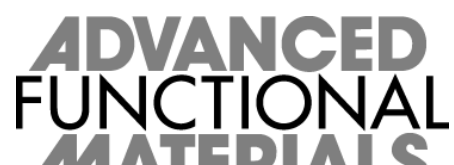

\title{
Morphological control for high performance, solution-processed planar heterojunction perovskite solar cells
}

By Giles E. Eperon, Victor M. Burlakov, Pablo Docampo, Alain Goriely, and Henry J. Snaith*

[*] Dr. H. J. Snaith, Giles E. Eperon, Dr. Pablo Docampo

Department of Physics, University of Oxford, Clarendon Laboratory, Parks Road, Oxford OX1 3PU, UK

E-mail: h.snaith1@physics.ox.ac.uk

Dr. Victor M. Burlakov, Prof. Alain Goriely

Mathematical Institute, OCCAM, University of Oxford, 24-29 St Giles', Oxford OX1 3LB, UK

Keywords: perovskite, solar cell, morphology, high efficiency, photovoltaics, solution processed

Organometal trihalide perovskite based solar cells have exhibited the highest efficiencies todate when incorporated into mesostructured composites. However, thin solid films of a perovskite absorber should be capable of operating at the highest efficiency in a simple planar heterojunction configuration. Here, we show that film morphology is a critical issue in planar heterojunction $\mathrm{CH}_{3} \mathrm{NH}_{3} \mathrm{PbI}_{3-\mathrm{x}} \mathrm{Cl}_{\mathrm{x}}$ solar cells. We carefully control the morphology by varying processing conditions, and demonstrate that the highest photocurrents are attainable only with the highest perovskite surface coverages. With optimized solution based film formation we achieve power conversion efficiencies of up to $11.4 \%$, the first report of efficiencies above $10 \%$ in fully thin-film solution processed perovskite solar cells with no mesoporous layer. 


\section{Introduction}

Submitted to

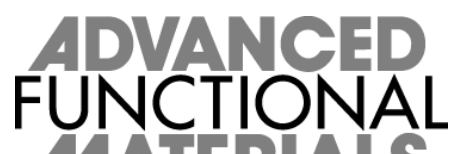

Thin-film solar cells are an important technology, promising cost-competitive solar power via reduced material and fabrication costs as compared to the established crystalline silicon industry. Such systems make use of the high absorption coefficients of direct bandgap semiconductors to allow films of a few microns or less to absorb most incident sunlight across their bandwidth, as opposed to the hundreds of microns typically found in wafer based $\mathrm{Si}$ solar cells. ${ }^{[1]}$ High efficiencies, approaching those of crystalline silicon, have been attained for cadmium telluride (CdTe) and other compound absorbers; ${ }^{[2]}$ however, fabrication of the best performing cells relies on vacuum deposition and/or high-temperature processes, pushing potential production costs higher and limiting substrate choice. ${ }^{[3]}$ The highest performing thinfilm technologies, namely CdTe and copper indium gallium (di)selenide (CIGS), additionally include elements which are in limited supply and are likely to bottleneck Terawatt scale solar energy production. ${ }^{[4-6]}$ Solution-processing represents the lowest-cost production method for thin-film solar cells. Cells can be fabricated via spin-coating, blade-coating, spraying, inkjet printing, gravure printing or slot-dye coating. ${ }^{[3,7]}$ The highest efficiency solution-processed thin-film solar cells, reaching efficiencies of over $11 \%$, have been achieved with solutionprocessable copper zinc tin chalcogenides (CZTS/Se) and CIGS bulk inorganics. ${ }^{[8,9]}$ However, the production of these solar cells relies on the use of toxic hydrazine and a high-temperature sintering process. If devices can be fully fabricated without the need for high-temperature annealing steps, greater versatility of substrate choice exists and costs of processing and infrastructure required for manufacture could be considerably reduced. Dye-sensitized solar cells, ${ }^{[10]}$ quantum dot solar cells, ${ }^{[11]}$ and organic solar cells ${ }^{[12]}$ can be fabricated in this way, but due to fundamental energy losses associated with charge separation in a low dielectric or energetically disordered medium their theoretical maximum performances fall below those of inorganic thin-film solar cells. ${ }^{[13,14]}$ 
Recently, a new family of solution-processable semiconducting perovskite structured materials based on organolead trihalide compounds have been developed, resulting in very effective sensitizers in hybrid solid-state solar cells, with published efficiencies of up to $12.3 \% .^{[15-18]}$ More importantly, they have been shown to exhibit ambipolar transport, allowing them to replace the hole or electron transporter in hybrid cells, ${ }^{[19-21]}$ making this family of materials suitable for solution-processable thin-film solar cells. ${ }^{[15-19,21-23]}$ Particularly, the perovskite $\mathrm{CH}_{3} \mathrm{NH}_{3} \mathrm{PbI}_{3-\mathrm{x}} \mathrm{Cl}_{\mathrm{x}}$ has been demonstrated to function in a thinfilm architecture, with a layer of bulk crystalline perovskite formed over a mesoporous alumina scaffold. ${ }^{[15]}$ In previous work, planar perovskite $p-i-n$ heterojunctions with no mesoporous layer gave power conversion efficiencies of up to $4.9 \%$, while the highest power conversion efficiencies of up to $12.3 \%$ were shown in a 'meso-superstructured' solar cell (MSSC) configuration with the perovksite fully infiltrating a porous alumina scaffold. However, internal quantum efficiencies of almost $100 \%$ for the planar configuration pointed towards its promise as an ultimately more efficient architecture. ${ }^{[15]}$ It is also beneficial from a production point of view to simplify the cell architecture. Hence, high efficiency cells with simply a single solution processed solid absorber layer would be advantageous.

It has been proposed that the planar thin-film architecture's lower performance may arise from pin-hole formation, incomplete coverage of the perovskite resulting in low-resistance shunting paths and lost light absorption in the solar cell; as in other technologies the issue of film formation is likely to be extremely important in the planar junction. ${ }^{[24-26]}$ It is wellknown that as-fabricated thin films are often thermodynamically unstable, and likely to dewet or agglomerate upon annealing, as predicted from energetic considerations. ${ }^{[27]}$ By following the previously reported fabrication protocol for perovskite solution coating on flat substrates, we observe significant dewetting leading to incomplete coverage and non-uniform film thickness. With optimised film formation, primarily controlling the atmosphere, annealing 
Submitted to
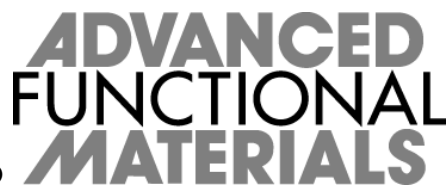

temperature and film thickness, we are able, for the first time, to form via solution casting uniform thin perovskite films with "full coverage" with no mesoporous layer. Doing so, we more than double the previously reported maximum power conversion efficiency in this configuration, achieving values of up to $11.4 \%$ in $\mathrm{CH}_{3} \mathrm{NH}_{3} \mathrm{PbI}_{3-\mathrm{x}} \mathrm{Cl}_{\mathrm{x}}$ planar heterojunction solar cells. This matches the best performing hydrazine processed CZTSSe thin film solar cell, ${ }^{[8]}$ and represents the first report of over $10 \%$ efficiency in this new fully thin-film solution processed perovskite technology.

\section{Results and discussion}

The perovskite MSSCs studied are effectively a distributed heterojunction. The $\mathrm{CH}_{3} \mathrm{NH}_{3} \mathrm{PbI}_{3-}$ ${ }_{\mathrm{x}} \mathrm{Cl}_{\mathrm{x}}$ perovskite, infiltrated within an alumina scaffold, acts as the intrinsic absorber and electron transporter, and 2,2',7,7'-tetrakis-(N,N-di-p-methoxyphenylamine)9,9'spirobifluorene (spiro-OMeTAD) as the p-type hole transporter. Previously, the highest efficiencies have been obtained in this "infiltrated" architecture. ${ }^{[15,23]}$ A typical MSSC cell would have short-circuit current $\left(\mathrm{J}_{\mathrm{sc}}\right)$ of $17-20 \mathrm{mAcm}^{-2}$, open-circuit voltage $\left(\mathrm{V}_{\mathrm{oc}}\right)$ of $1.0-1.1 \mathrm{~V}$, and fill factor of 0.6-0.7, which combine to result in power conversion efficiencies of above $10 \% .{ }^{[28]}$ In the planar heterojunction configuration, illustrated in Fig. 1a, $\mathbf{J}_{\mathrm{sc}}, \mathrm{V}_{\mathrm{oc}}$, and fill factor are lower, as shown in the current voltage curve presented in Fig. 1b. The significant drop in these parameters may be a result of poor coverage of perovskite films. The effects of poor coverage are twofold: Firstly, if there are regions of no perovskite coverage, light will pass straight through without absorption, decreasing the available photocurrent; secondly, insufficient coverage results in a high frequency of "shunt paths" allowing contact between spiro-OMeTAD and the $\mathrm{TiO}_{2}$ compact layer. Any such contact will act as a parallel diode in the solar cell equivalent circuit, causing a drop in $\mathrm{V}_{\mathrm{oc}}$ and fill factor, and accordingly power conversion efficiency. ${ }^{[29,30]}$

To investigate whether surface coverage is indeed an issue in this type of solar cell, we took scanning electron microscope (SEM) images of the surface morphology of perovskite films 
Submitted to 14 A

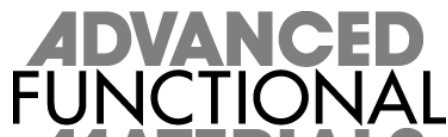

with increasingly reduced thickness of mesoporous $\mathrm{Al}_{2} \mathrm{O}_{3}$, transitioning from the MSSC infiltrated configuration to the thin-film planar heterojunction. These SEM images are shown in Fig. 2, and the samples were prepared in air according to the published procedure. As previously shown, ${ }^{[15]}$ we see that in addition to perovskite crystallization within the mesoporous layer, a perovskite "capping layer" is also formed. The surface coverage of the capping layer is not complete however, and increases with decreasing alumina thickness (Fig. 2). However when the alumina is fully removed, the perovskite film forms differently, as seen in Fig 2. We can estimate the fractional surface coverage of perovskite, by simply setting a threshold on the image and calculating the area above and below threshold. For the films with no mesoporous layer, the surface coverage unexpectedly drops to values of $\sim 75 \%$, indicating that one of the main roles of the alumina layer is to control film formation in such a way as to produce a high coverage capping layer. When no alumina is present, the reduced perovskite coverage likely leads to the reduced $\mathrm{J}_{\mathrm{sc}}, \mathrm{V}_{\mathrm{oc}}$ and fill factor as suggested above. If this coverage could be increased, we would expect higher performances, possibly exceeding the MSSC structure.

To understand why these voids are present in the perovskite layers coated on flat films, we studied a time-series of the perovskite anneal process, with the SEM images shown in Fig. 2. Since films are extremely moisture-sensitive until fully crystallized, here we processed the films in a dry nitrogen-filled glovebox to enable characterisation of the pre-crystalized films. We notice rapid degradation of non-annealed films in moist atmospheres, likely due to the hygroscopicity of the methylammonium cation. ${ }^{[16]}$ Straight after spin-coating, film coverage is high, with a number of small pores. Upon annealing, many additional small pores form rapidly (Fig. 2, 10mins), and then either increase in size or close up until the final crystalline phase is reached (Fig. 2, 60 mins). Upon formation of stable crystals, we observe pore evolution to cease, likely due to evaporation and mass transport no longer being energetically 
Submitted to

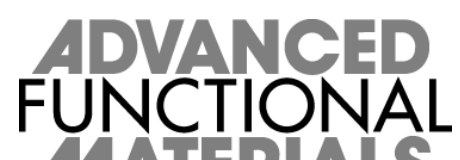

favourable. The morphology of samples prepared in an inert atmosphere is notably different from the air-processed samples, likely due to the lack of moisture, which otherwise attacks the surface as the film forms. We suggest that the change in the film morphology upon annealing is driven by surface energy minimisation and is facilitated by mass loss. ${ }^{[27]}$ A precursor solution with an excess of methylammonium and halide compared to the lead content is used, and as such we propose then that upon spin-coating, an organic and halide-rich film is formed. As the thermal annealing process takes place, it is likely to be energetically favourable for the excess organic and halide to evaporate, once a temperature threshold is reached. This would continue until a crystal with equimolar amounts of organic, metal and halide (1:1:3 organic:metal:halide by moles) is left. Once crystallized, mass loss ceases since a low-energy state has been reached.

Depending on the conditions, we have observed the pores present in a film to either in general increase or decrease in size. Minimisation of surface energy of pores is a non-trivial problem, but broadly speaking would depend upon the interaction energies of the perovskite and air, and the perovskite and the substrate. The final crystalline morphology would depend on the dynamics of annealing, which would in turn depend on anneal temperature, solvent and mass evaporation and transport rate, and film thickness. Elsewhere, we will present a full study and mathematically model of the complex dewetting process of such solution-cast perovskite films.

Here, we form films on a fluorine-doped tin oxide (FTO) covered glass substrate coated with a $\mathrm{TiO}_{2}$ compact layer. We can easily control film thickness and anneal temperature; filmsubstrate interaction energy and solvent evaporation rate are more complex to vary and quantify and so are held fixed. We vary the temperature and initial thickness, and measure the resulting crystallized perovskite coverage using the image analysis software Image ${ }^{[31]}$. 
Submitted to 14 ATERAS

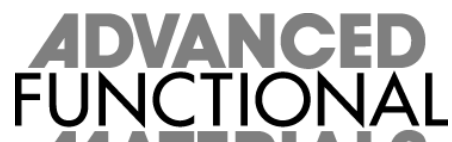

SEM images of representative perovskite films are shown in Fig. 3a, and the calculated coverages are plotted in Fig. $3 b$ and Fig. 3c. The effect of varying temperature on the wetting of the thin film is shown in (a). As anneal temperature increases, the number of pores in the final film decreases, but their size increases and the morphology transitions from continuous layers into discrete islands of perovskite. This has the effect of reducing surface coverage, as seen in (b). Previously, annealing has been carried out at $100^{\circ} \mathrm{C}$; however this data suggests as low a temperature as possible should be used to attain maximum coverage whilst still enabling full crystallisation of the perovskite absorber.

The influence of thickness variation whilst holding the temperature fixed at $95^{\circ} \mathrm{C}$ is also shown in Fig 3. We see that with increasing initial film thickness, the average pore size increases, though there are fewer pores per unit area. The effect on coverage is thus not obvious; image analysis reveals that thicker initial films result in marginally greater coverages, as seen in Fig. 3b. The previous standard protocol used a thickness of around 500-700nm. This is within the expected region of high coverage; the primary factors of importance when understanding the photovoltaic behaviour for these perovskite films of $>200 \mathrm{~nm}$ thickness becomes a balance between full photon absorption and electron and hole diffusion length throughout the bulk perovskite. The detailed study of charge dynamics is beyond the scope of this report, but an experimental optimisation (not detailed herein) of devices suggested thicknesses between 400 and 800nm were suitable for attaining high efficiency devices.

Knowing the effect of these parameters, we are able to tune the desired perovskite coverage, though $100 \%$ coverage was not attainable by varying initial film thickness and temperature of anneal. To establish if increasing the perovskite coverage solves the decreases in performance seen previously, we fabricated planar heterojunction devices with a range of perovskite coverages. We varied anneal temperature, and additionally varied the solvent used to obtain 
Submitted to
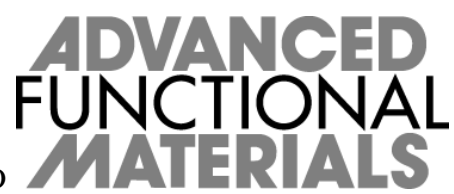

the lowest coverages. Employing more slowly-evaporating solvents, dimethylsulfoxide and nmethyl-2-pyrrolidone, instead of DMF, reduces the surface coverage.

Mean device parameters for a single batch of devices, extracted from current-voltage curves under simulated AM1.5, $100 \mathrm{mWcm}^{-2}$ sunlight are shown in Fig. 4. Short-circuit photocurrent shows a clear trend with coverage. At coverages of $\sim 56 \%$, average $\mathrm{J}_{\mathrm{sc}}$ is around $11 \mathrm{mAcm}^{-2}$. As the coverage increases up to $\sim 94 \%, \mathrm{~J}_{\mathrm{sc}}$ increases linearly, up to average values of around $18 \mathrm{mAcm}^{-2}$. The best performing individual cells show $\mathrm{J}_{\mathrm{sc}}$ above $21 \mathrm{mAcm}^{-2}$, matching the highest currents reported in the perovskite solar cells to date. The effect of coverage on power conversion efficiency, shown in Fig 4(b), is not so clear. Despite the trend in photocurrent, fill factor and $\mathrm{V}_{\mathrm{oc}}$ do not follow easily understandable trends with coverage (see SI Fig. S2). It is likely that the changing morphology of the film additionally results in varying electronic and physical contact between hole transporter--perovskite, hole transporter- $\mathrm{TiO}_{2}$ layer and perovskite- $\mathrm{TiO}_{2}$ layer, which complicates the situation. However, the lowest average efficiencies are observed for the lowest coverages, and the highest average efficiencies are observed for the highest coverages. Though the intermediate behaviour is not clear, our study supports the logical conclusion that high coverage is the optimum configuration for high power generation.

Motivated by this simple principle, and given that we were unable to attain $100 \%$ coverage by optimization of temperature and thickness alone, we attempted to achieve full coverage by varying the film-substrate interaction energy. This was achieved by altering the thickness of the $\mathrm{TiO}_{2}$ compact layer. Indeed, we observed that by using thicker $\mathrm{TiO}_{2}$ compact layers, increased coverage was attained. Representative films are shown in Fig. 5. This discovery enabled us to produce full-coverage perovskite films. We currently propose that the n-type compact layer interacts electronically with the perovskite film during formation; possibly a thicker layer is able to transfer more electronic charge to the perovskite assisting its formation 


\section{Submitted to}

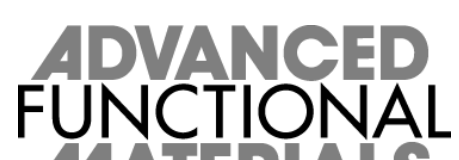

near the surface due to differing electrostatics. Further investigation of this important effect is currently in progress.

We fabricated devices from a range of $\mathrm{TiO}_{2}$ compact layer thicknesses, measuring perovskite coverage and device parameters to determine if any additional gains in efficiency are seen compared to $90 \%+$ coverage. $\mathrm{TiO}_{2}$ compact layer thickness was varied by repeatedly spincoating more layers of the $\mathrm{TiO}_{2}$ precursor solution. A single spin-coated layer was measured to be approximately $75 \mathrm{~nm}$ thick. Fig. $5 \mathrm{~d}$ shows the dependence of perovskite coverage, $\mathrm{J}_{\mathrm{sc}}$, power conversion efficiency and $\mathrm{V}_{\text {oc }}$ on increased $\mathrm{TiO}_{2}$ compact layer thickness. With thicker $\mathrm{TiO}_{2}$ layers, an increase in perovskite coverage is seen; however, disappointingly $\mathrm{J}_{\mathrm{sc}}$ and $\mathrm{V}_{\mathrm{oc}}$ both drop, resulting in lower device efficiencies. $\mathrm{A} \mathrm{TiO}_{2}$ compact layer of increased thickness is likely to have a significant effect upon device performance since the relatively resistive, and possibly depleted $\mathrm{TiO}_{2}$ layer is critical to electron collection. Thick $\mathrm{TiO}_{2}$ compact layers have been shown previously to hinder charge extraction in dye-sensitized solar cells due to increased series resistance; ${ }^{[32]}$ this is likely the reason for the observed decrease in performance.

Despite not achieving further improvements in efficiency with full coverage based on thicker $\mathrm{TiO}_{2}$ compact layers, due to series resistance limitations, we obtained impressive efficiencies with the highest perovskite coverages on the thinnest $\mathrm{TiO}_{2}$ layer. This was achieved by annealing at a lower temperature of $90^{\circ} \mathrm{C}$, with an initial perovskite film thickness of 450 550nm. The improvement in average current-voltage characteristics, and hence overall performance resulting from this process is shown in Fig 6a. The current-voltage curve corresponding to the most efficient device measured is shown in Fig 6b. The efficiency of the most efficient device is $11.4 \%$, which represents greater than a two-fold improvement over the previous report of solution processed planar heterojunction perovskite solar cells. ${ }^{[15]}$ Improvements are due to increased $\mathrm{J}_{\mathrm{sc}}, \mathrm{V}_{\mathrm{oc}}$ and fill factor, and are likely to stem from two 
Submitted to 14 A R

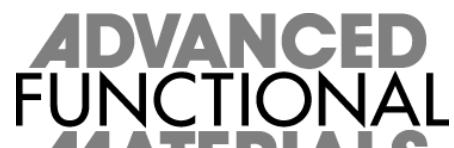

effects resulting from improved perovskite coverage. Firstly, it has enabled collection of a higher fraction of incident photons, increasing useful current generated. Secondly, the increased coverage has reduced contact area between the hole transporter and the holeblocking layer, which removes a shunt path previously leading to leakage currents. ${ }^{[33]}$ Reduction of these shunt paths would therefore be expected to enhance the fill factor and $V_{o c}$ as has been observed in the work presented here. Full elimination of shunt paths may be expected to increase $\mathrm{V}_{\text {oc }}$ up to levels seen in the MSSC configuration ( 1.1V). We expect that further tuning of the interaction energy between the $\mathrm{TiO}_{2}$ compact layer and the perovskite, whilst still employing thin $\mathrm{TiO}_{2}$ films, will enable $100 \%$ perovskite coverage upon electronically optimal layers, resulting in further enhanced performance.

\section{Conclusions}

By understanding and controlling morphology of perovskite films originating from a nonstoichiometric composition of precursor salts, we have demonstrated the critical role of uniform perovskite film formation in planar heterojunction perovskite solar cells. The highest efficiencies are achievable only with the highest surface coverages. We have fabricated flat heterojunction cells at low temperatures with efficiencies of up to $11.4 \%$, the first report of a fully thin-film solution processed perovskite solar cell with no mesoporous layer with efficiency above $10 \%$. This indicates that a mesoporous layer is no longer necessary to achieve high efficiency perovskite cells. Simplification of the cell architecture in this way increases the versatility of such cells, and can enable easier and cheaper manufacturing on a large scale. There is immense scope for further research to lead to even higher efficiency planar heterojunction perovskite cells.

\section{Experimental}

Perovskite precursor preparation: Methylamine iodide (MAI) was prepared by reacting methylamine, $33 \mathrm{wt} \%$ in ethanol (Sigma-Aldrich), with hydroiodic acid (HI) $57 \mathrm{wt} \%$ in water 
Submitted to

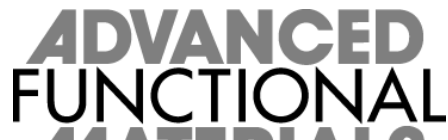

14ATRAS

(Sigma-Aldrich), at room temperature. HI was added dropwise while stirring. Upon drying at $100^{\circ} \mathrm{C}$, a white powder was formed, which was dried overnight in a vacuum oven before use. To form the non-stoichiometric $\mathrm{CH}_{3} \mathrm{NH}_{3} \mathrm{PbI}_{3-\mathrm{x}} \mathrm{Cl}_{\mathrm{x}}$ precursor solution, methylammonium iodide and lead (II) chloride (Sigma-Aldrich) are dissolved in anhydrous $N, N$ Dimethylformamide (DMF), dimethylsulfoxide (DMSO), or n-methyl-2-pyrrolidone (NMP) at a 3:1 molar ratio of MAI to $\mathrm{PbCl}_{2}$, with final concentrations $0.88 \mathrm{M}$ lead chloride and 2.64M methylammonium iodide. This solution is stored under a dry nitrogen atmosphere. As shown in Figure S1, the X-ray diffraction data from this perovskite shows a highly crystalline material with peaks as in the previously reported data. ${ }^{[15,20]}$

Substrate preparation: Devices were fabricated on fluorine-doped tin oxide (FTO) coated glass (Pilkington, $7 \Omega \square^{-1}$ ). Initially FTO was removed from regions under the anode contact, to prevent shunting upon contact with measurement pins, by etching the FTO with $2 \mathrm{M} \mathrm{HCl}$ and zinc powder. Substrates were then cleaned sequentially in $2 \%$ hallmanex detergent, acetone, propan-2-ol and oxygen plasma. A hole-blocking layer of compact $\mathrm{TiO}_{2}$ was deposited by spin-coating a mildly acidic solution of titanium isopropoxide in ethanol, and annealed at $500^{\circ} \mathrm{C}$ for 30 minutes. Spin-coating was carried out at 2000rpm for 60 seconds.

Perovskite solar cell fabrication: To form the perovskite layer, the non-stoichiometric precursor was spin-coated on the substrate in a nitrogen-filled glovebox, at 2000rpm for 45 seconds. To vary the initial layer thickness, the precursor was diluted in DMF, or the spin speed varied. After spin-coating, the films were left to dry at room temperature in the glovebox for 30 minutes, to allow slow solvent evaporation. They were then annealed on a hotplate in the glovebox at $90^{\circ} \mathrm{C}, 110^{\circ} \mathrm{C}, 130^{\circ} \mathrm{C}, 150^{\circ} \mathrm{C}$ or $170^{\circ} \mathrm{C}$, for $120,50,20,10$, or 7.5 minutes respectively.

A hole-transporting layer was then deposited in air via spin-coating a $0.79 \mathrm{M}$ solution of 2,2',7,7'-tetrakis-(N,N-di-p-methoxyphenylamine)9,9'-spirobifluorene (spiro-OMeTAD) in 
Submitted to

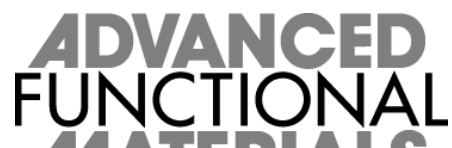

chlorobenzene, with additives of lithium bis(trifluoromethanesulfonyl)imide and 4-tertbutylpyridine. Spin-coating was carried out at 2000rpm for 60 seconds. Devices were then left overnight in air for the spiro-OMeTAD to dope via oxidation. ${ }^{[34]}$ Finally, 60nm gold electrodes were thermally evaporated under vacuum of $\sim 10^{-6}$ Torr, at a rate of $\sim 0.1 \mathrm{~nm} / \mathrm{s}$, to complete the devices.

Materials characterization: A field emission scanning electron microscope (Hitachi S-4300) was used to acquire SEM images. To acquire images of moisture-sensitive unannealed perovskite films, samples were kept in nitrogen atmosphere until imaging. To determine coverage of perovskite films from SEM images, Image ${ }^{[30]}$ was used to define a greyscale threshold such that the perovskite was distinct from the substrate, and percentage coverage was then calculated by the program. Sample thicknesses were measured using a Veeco Dektak 150 surface profilometer. X-ray diffraction (XRD) spectra were obtained from full devices with no evaporated electrodes, using a Panalytical X'Pert Pro x-ray diffractometer.

Solar cell characterization: The current density-voltage (J-V) curves were measured (2400 Series SourceMeter, Keithley Instruments) under simulated AM 1.5 sunlight at $100 \mathrm{mWcm}^{-2}$ irradiance generated by an Abet Class AAB sun 2000 simulator, with the intensity calibrated with an NREL calibrated KG5 filtered Si reference cell. The mismatch factor was calculated to be less than $1 \%$. The solar cells were masked with a metal aperture to define the active area, typically $0.09 \mathrm{~cm}^{-2}$ (measured individually for each mask) and measured in a light-tight sample holder to minimize any edge effects and ensure that the reference cell and test cell are located during measurement in the same spot under the solar simulator.

\section{Acknowledgements}

This work was supported by EPSRC and Oxford Photovoltaics Ltd. through a Nanotechnology KTN CASE award, the European Research Council (ERC) HYPER PROJECT no. 279881. This publication is based in part upon work supported by Award No. KUK-C1-013-04, made by King Abdullah University of Science and Technology (KAUST). 
Submitted to

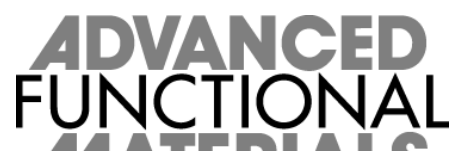

AG is a Wolfson/Royal Society Merit Award Holder and acknowledges support from a Reintegration Grant under EC Framework VII. VB is an Oxford Martin School Fellow and this work was in part supported by the Oxford Martin School. We would like to thank Edward Crossland and James Ball for valuable discussions.

Supporting Information is available online from Wiley InterScience or from the author.

Received: ((will be filled in by the editorial staff))

Revised: ((will be filled in by the editorial staff))

Published online: ((will be filled in by the editorial staff))

[1] D. B. Mitzi, O. Gunawan, T. K. Todorov, K. Wang, S. Guha, Solar Energy Materials and Solar Cells 2011, 95, 1421.

[2] M. A. Green, K. Emery, Y. Hishikawa, W. Warta, E. D. Dunlop, Progress in Photovoltaics: Research and Applications 2013, 21, 1.

[3] M. Grätzel, R. a J. Janssen, D. B. Mitzi, E. H. Sargent, Nature 2012, 488, 304.

[4] A. Bosio, D. Menossi, S. Mazzamuto, N. Romeo, Thin Solid Films 2011, 519, 7522.

[5] R. N. Bhattacharya, M. a. Contreras, B. Egaas, R. N. Noufi, A. Kanevce, J. R. Sites, Applied Physics Letters 2006, 89, 253503.

[6] V. Fthenakis, Renewable and Sustainable Energy Reviews 2009, 13, 2746.

[7] F. C. Krebs, Solar Energy Materials and Solar Cells 2009, 93, 394.

[8] D. A. R. Barkhouse, O. Gunawan, T. Gokmen, T. K. Todorov, D. B. Mitzi, Progress in Photovoltaics: Research and Applications 2012, 20, 6.

[9] T. K. Todorov, O. Gunawan, T. Gokmen, D. B. Mitzi, Progress in Photovoltaics: Research and Applications 2013, 21, 82.

[10] M. K. Nazeeruddin, E. Baranoff, M. Grätzel, Solar Energy 2011, 85, 1172.

[11] E. H. Sargent, Nature Photonics 2012, 6, 133.

[12] J. Nelson, Materials Today 2011, 14, 462.

[13] H. J. Snaith, Advanced Functional Materials 2010, 20, 13. 
Submitted to

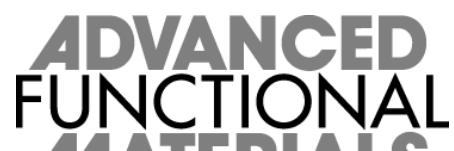

[14] T. Kirchartz, K. Taretto, U. Rau, The Journal of Physical Chemistry C 2009, 113, 17958.

[15] J. M. Ball, M. M. Lee, A. Hey, H. J. Snaith, Energy \& Environmental Science 2013, 6, 1739.

[16] J. H. Noh, S. H. Im, J. H. Heo, T. N. Mandal, S. Il Seok, Nano letters 2013, 13, 1764.

[17] H.-S. Kim, C.-R. Lee, J.-H. Im, K.-B. Lee, T. Moehl, A. Marchioro, S.-J. Moon, R. Humphry-Baker, J.-H. Yum, J. E. Moser, M. Grätzel, N.-G. Park, Scientific reports 2012, 2, 591.

[18] A. Kojima, K. Teshima, Y. Shirai, T. Miyasaka, Journal of the American Chemical Society 2009, 131, 6050.

[19] I. Chung, B. Lee, J. He, R. P. H. Chang, M. G. Kanatzidis, Nature 2012, 485, 486.

[20] M. M. Lee, J. Teuscher, T. Miyasaka, T. N. Murakami, H. J. Snaith, Science 2012, 338, 643.

[21] L. Etgar, P. Gao, Z. Xue, Q. Peng, A. K. Chandiran, B. Liu, M. K. Nazeeruddin, M. Grätzel, Journal of the American Chemical Society 2012, 134, 17396.

[22] J.-H. Im, C.-R. Lee, J.-W. Lee, S.-W. Park, N.-G. Park, Nanoscale 2011, 3, 4088.

[23] M. M. Lee, J. Teuscher, T. Miyasaka, T. N. Murakami, H. J. Snaith, Science 2012, 338, 643.

[24] X. Yang, J. Loos, Macromolecules 2007, 40, 1353.

[25] Z.-S. Wang, H. Kawauchi, T. Kashima, H. Arakawa, Coordination Chemistry Reviews 2004, 248, 1381.

[26] W. Jaegermann, A. Klein, T. Mayer, Advanced Materials 2009, 21, 4196.

[27] C. V. Thompson, Annual Review of Materials Research 2012, 42, 399.

[28] H. J. Snaith, Energy \& Environmental Science 2012, 5, 6513

[29] P. Docampo, H. J. Snaith, Nanotechnology 2011, 22, 225403. 
[30] L. Han, N. Koide, Y. Chiba, T. Mitate, Applied Physics Letters 2004, 84, 2433.

[31] W. Rasband, ImageJ 2005, http://rsb.info.nih.gov/ij/.

[32] B. Peng, G. Jungmann, C. Jäger, D. Haarer, H.-W. Schmidt, M. Thelakkat, Coordination Chemistry Reviews 2004, 248, 1479.

[33] H. J. Snaith, N. C. Greenham, R. H. Friend, Advanced Materials 2004, 16, 1640.

[34] A. Abate, T. Leijtens, S. Pathak, J. Teuscher, R. Avolio, M. E. Errico, J. Kirkpatrik, J.

M. Ball, P. Docampo, I. McPherson, H. J. Snaith, Physical Chemistry Chemical Physics 2013, $15,2572$. 


\section{FUNCTIONAL Submitted to MATERIALS}

a

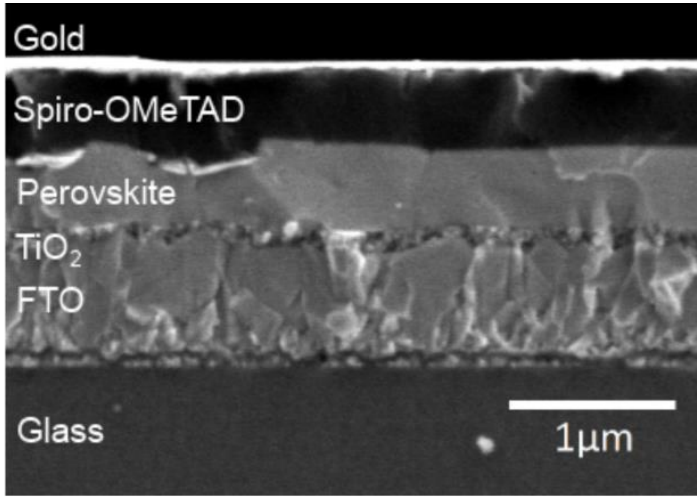

b

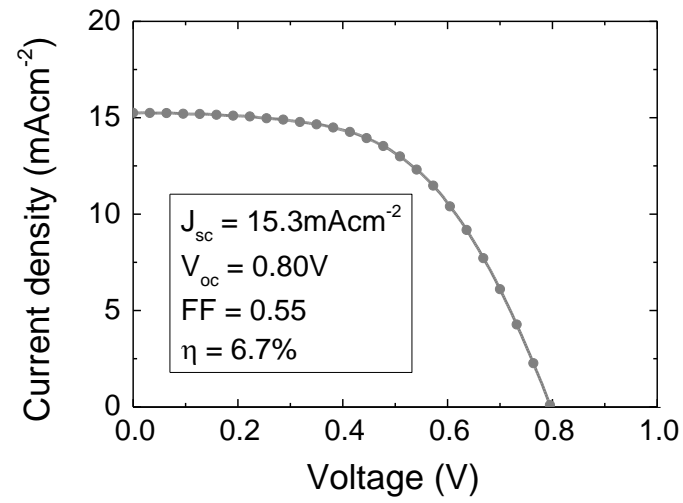

Figure 1. (a) Cross-sectional SEM micrograph showing device architecture of the planar heterojunction solar cells. (b) Average current-voltage characteristics from a batch of 10 nonoptimised planar heterojunction solar cells, prepared according to the published procedure, ${ }^{[15]}$ measured under simulated AM1.5 sunlight. We note that the presented JV curve is a numerical average of ten different JV curves, not simply a representative JV curve.
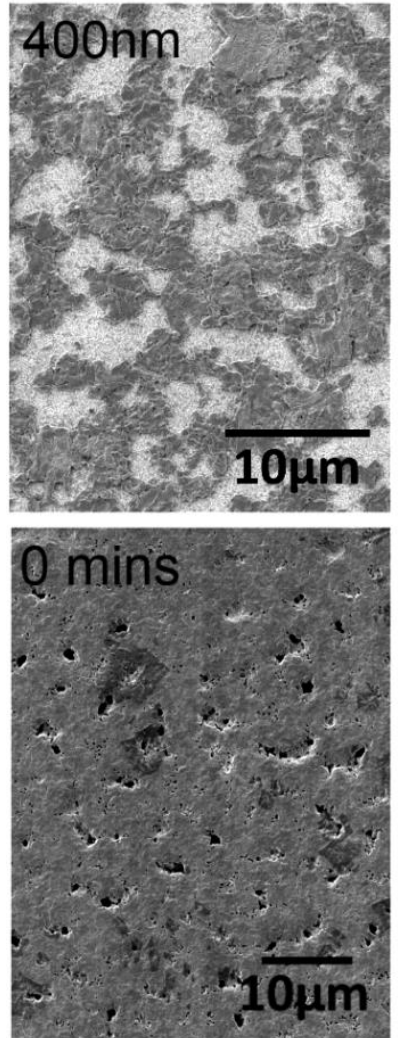
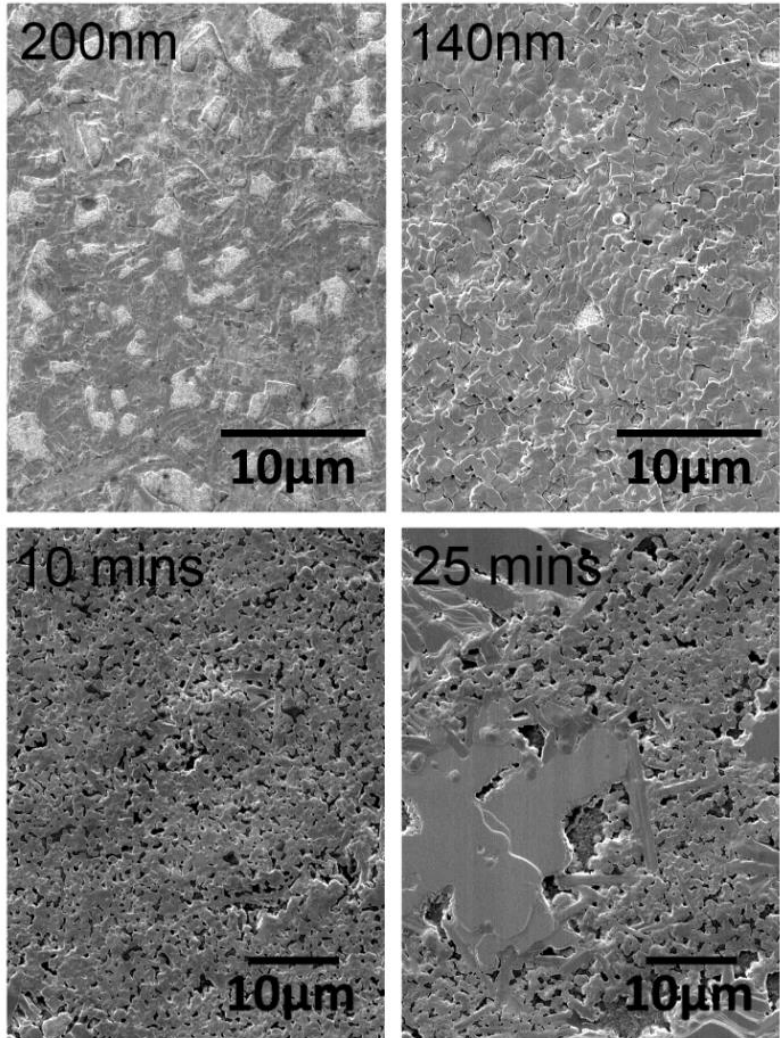

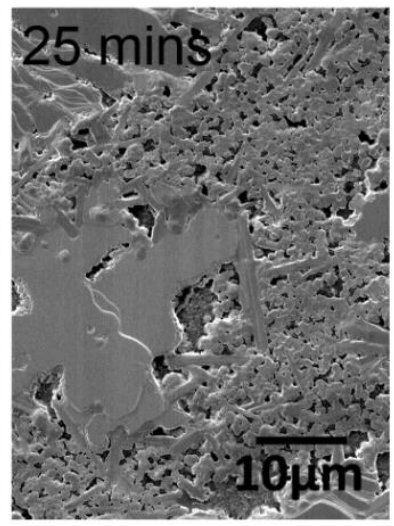

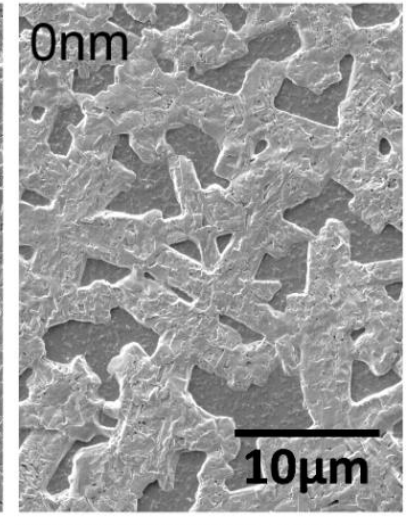

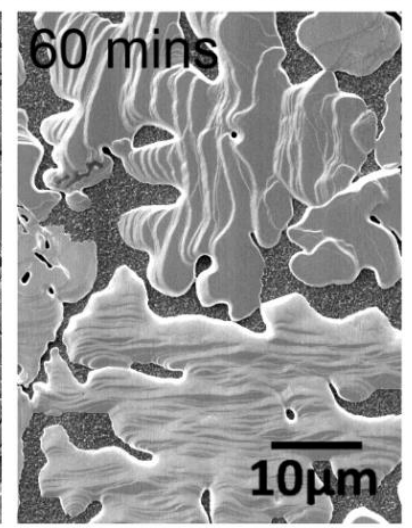




\section{Submitced to MATTENAIS}

Figure 2. Top row: SEM micrographs of the top surfaces of $\mathrm{CH}_{3} \mathrm{NH}_{3} \mathrm{PbI}_{3-\mathrm{x}} \mathrm{Cl}_{\mathrm{x}}$ films formed on alumina scaffolds of thickness shown. Bottom row: SEM micrographs of perovskite films on compact $\mathrm{TiO}_{2}$-coated FTO glass with $100^{\circ} \mathrm{C}$ anneal times shown on SEM images, prepared in a nitrogen atmosphere.

\section{a}
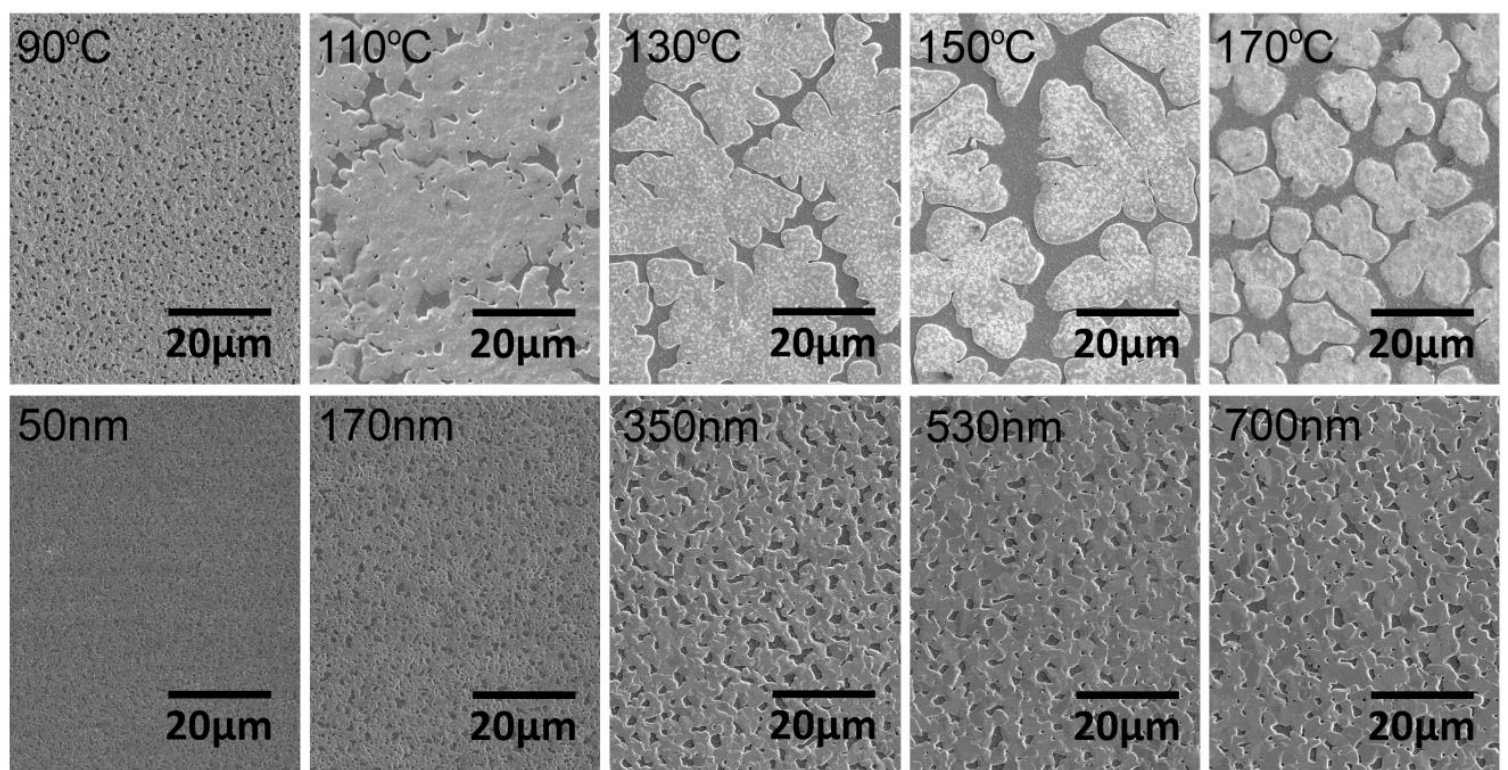

b

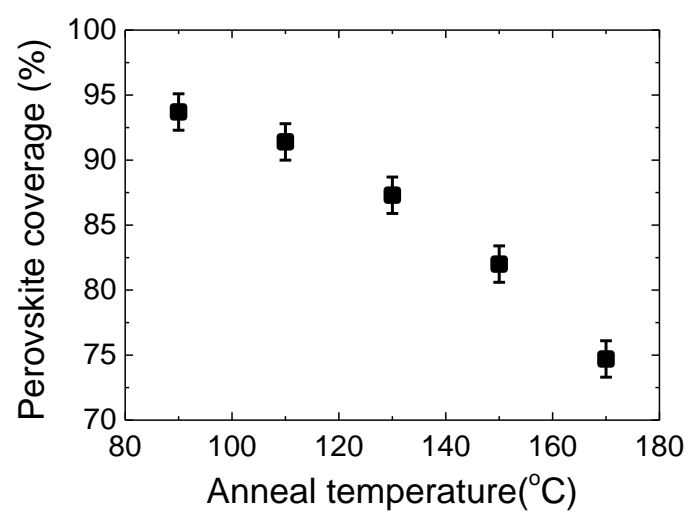

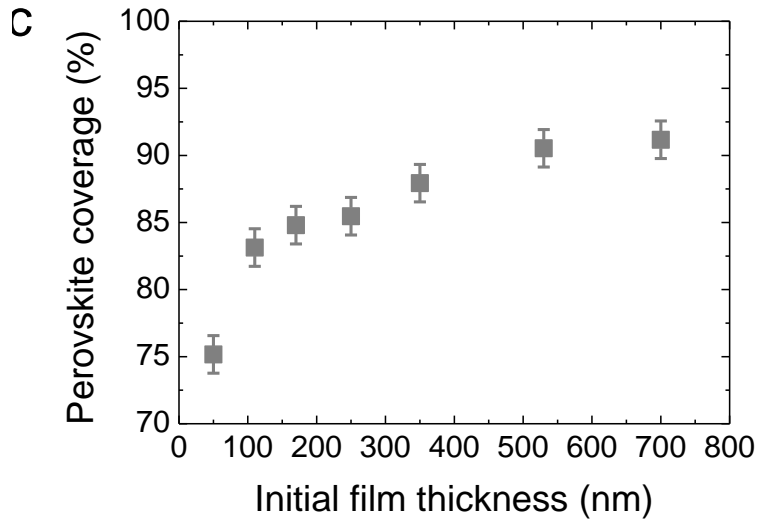

Figure 3. a) Top row: SEM micrographs showing dependence of perovskite coverage on annealing temperature, temperature shown on images, holding initial film thickness fixed at $650 \pm 50 \mathrm{~nm}$. Bottom row: effect of initial perovskite film thickness, shown on images, with 


\section{Submitted to FUNCTIONAL}

annealing temperature fixed at $95^{\circ} \mathrm{C}$. (b and c) Perovskite surface coverage as a function of (b) anneal temperature and (c) initial film thickness, calculated from SEM images.
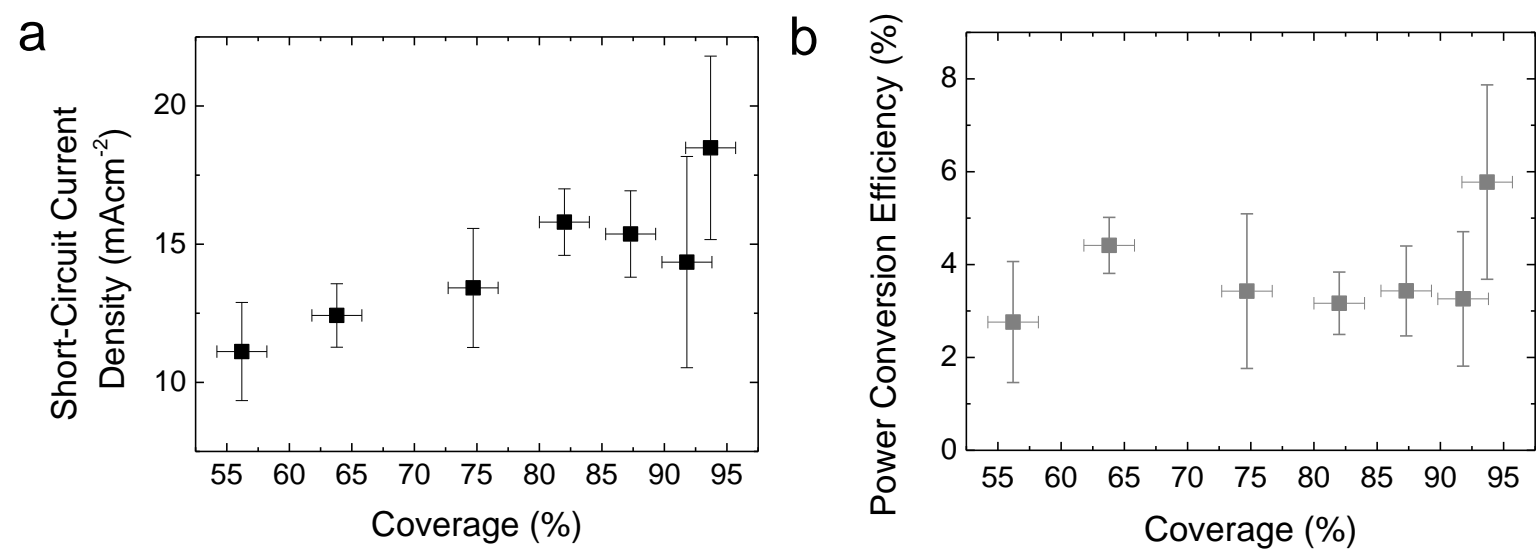

Figure 4. Dependence of the (a) short-circuit current density and (b) power conversion efficiency on perovskite coverage, extracted from solar cells illuminated under simulated AM1.5 sunlight of $100 \mathrm{mWcm}^{-2}$ irradiance. Each data point represents the mean from a set of 9 or more individual devices. 


\section{FUNCTIONAL
Submited to MATERIALS}
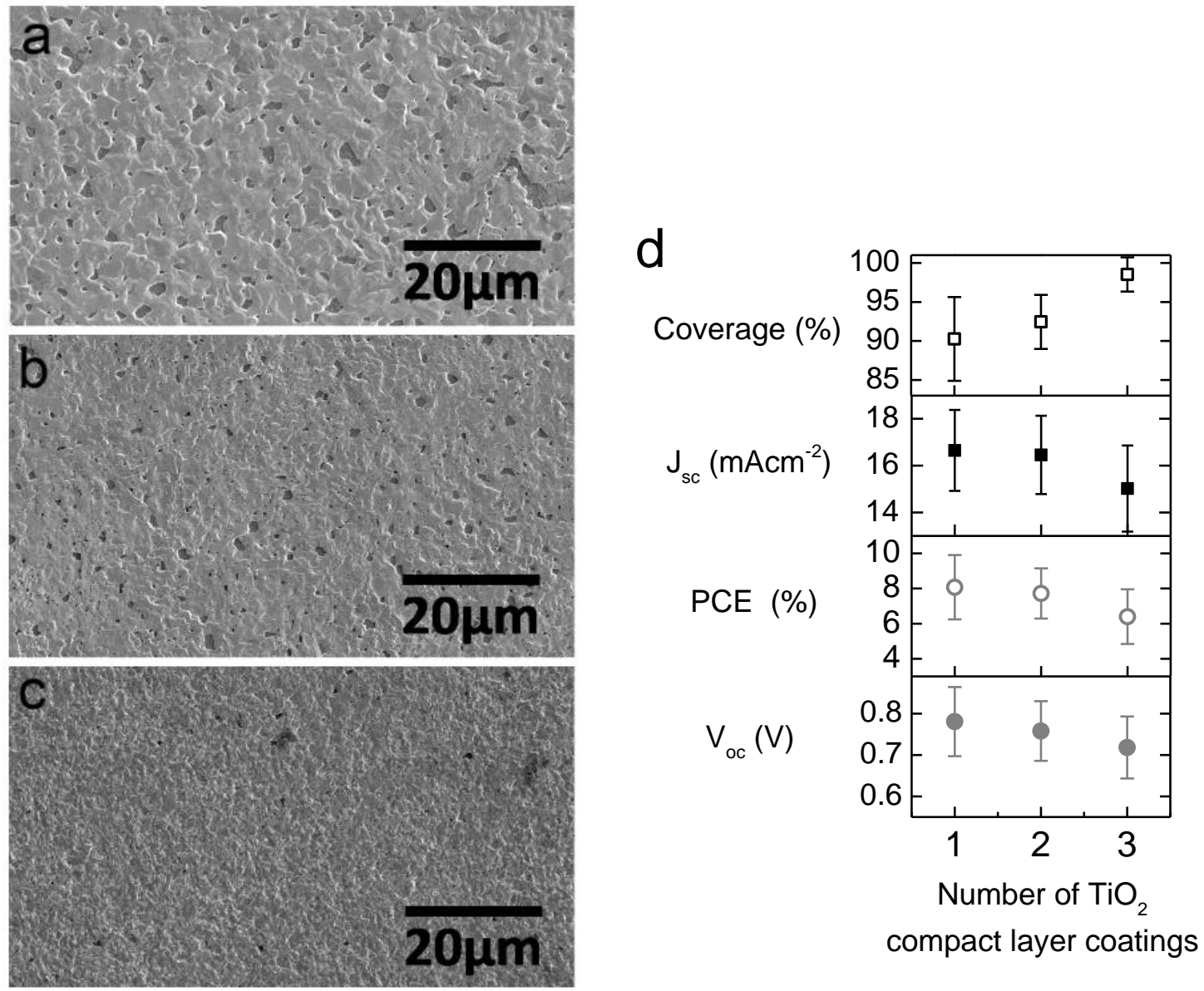

Figure 5. (a)-(c) SEM micrographs of perovskite films formed on (a) $\sim 75 \mathrm{~nm}$ (b) $\sim 150 \mathrm{~nm}$ and (c) $\sim 225 \mathrm{~nm}$ thick $\mathrm{TiO}_{2}$ compact layers coating FTO substrates. (d) Dependence of perovskite coverage and device parameters on the thickness of the $\mathrm{TiO}_{2}$ compact layers, in a single batch of devices. Each data point represents the mean from 32 or more individual devices, with the exception of coverage, which is based on 3 measurements per data point. 
Submitted to

\section{ADNANEED \\ MATERIALS}

a

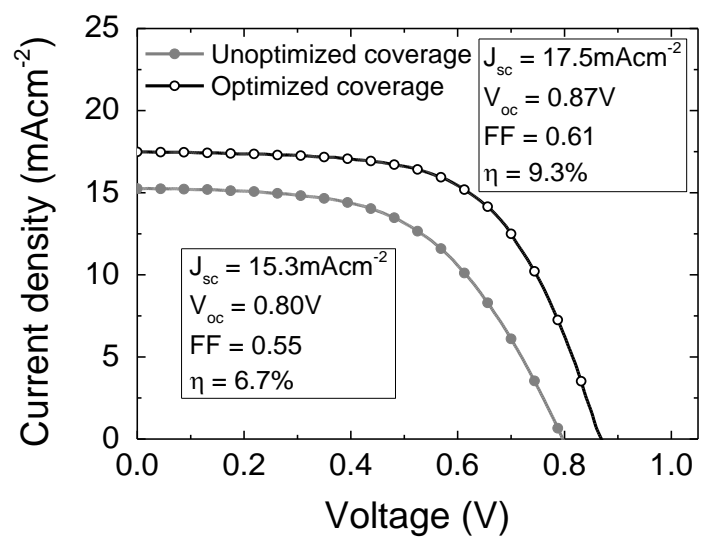

b

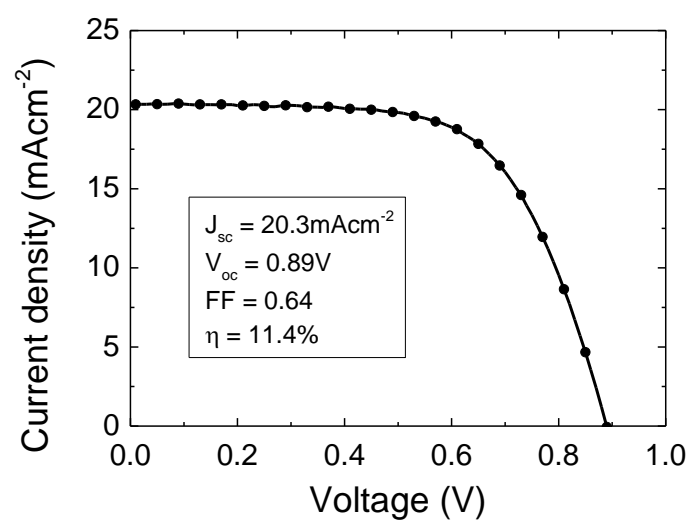

Figure 6. Current-voltage characteristics measured under simulated AM1.5 sunlight of (a) the average of a batch of 11 solar cells produced using the optimized high coverage planar heterojunction configuration, compared to the previously shown unoptimized batch and (b) the best performing solar cell based on the planar heterojunction configuration. 


\section{Table of contents entry}

Submitted to

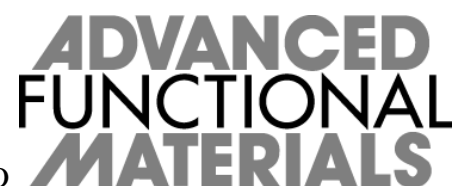

The critical role of perovskite morphology in planar heterojunction perovskite solar cells is probed and understood. Dewetting of perovskite films is minimized, to achieve uniform $100 \%$ coverage perovskite layers. Solution cast planar heterojunction solar cells with efficiencies of up to $11.4 \%$ are fabricated, a new record for such cells with no mesoporous layer.

Keywords: perovskite, morphology, high efficiency, photovoltaics, solution processed

Giles E. Eperon, Victor M. Burlakov, Pablo Docampo, Alain Goriely, and Henry J. Snaith*

Morphological control for high performance, solution-processed planar heterojunction perovskite solar cells

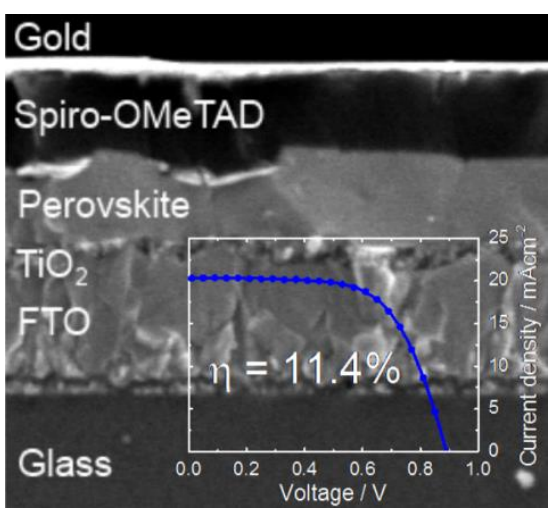


Supporting Information

Submitted to

1. Additional figures

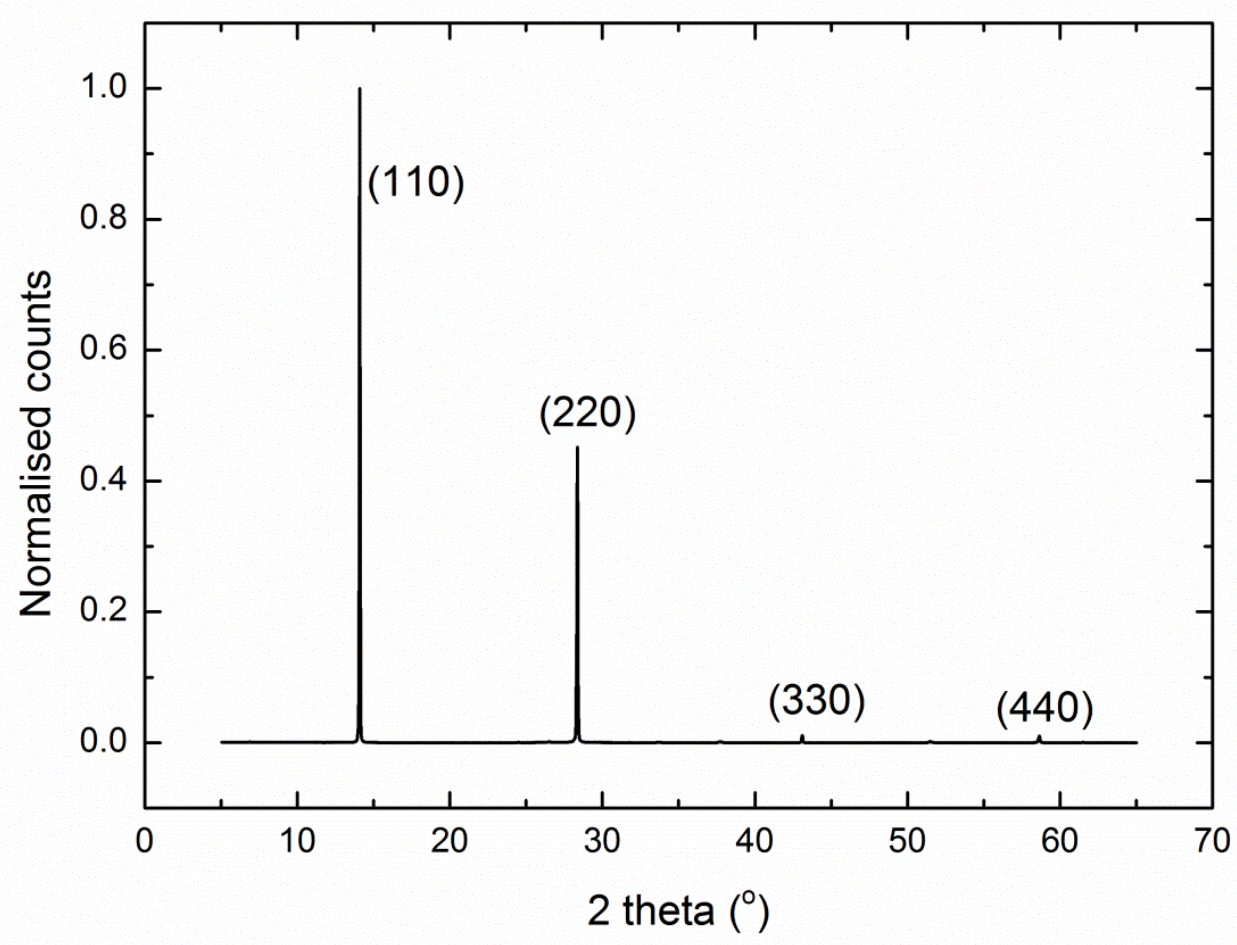

Figure S1. XRD spectrum of a high-coverage perovskite thin film prepared on a $\mathrm{TiO}_{2}$ compact layer coated FTO substrate, such as used in the optimized high-efficiency devices. Labelled peaks are assigned to the planes of a tetragonal perovskite lattice with $a=b=8.85 \AA$, $\mathrm{c}=12.73 \AA$. 
Submitted to 14 A

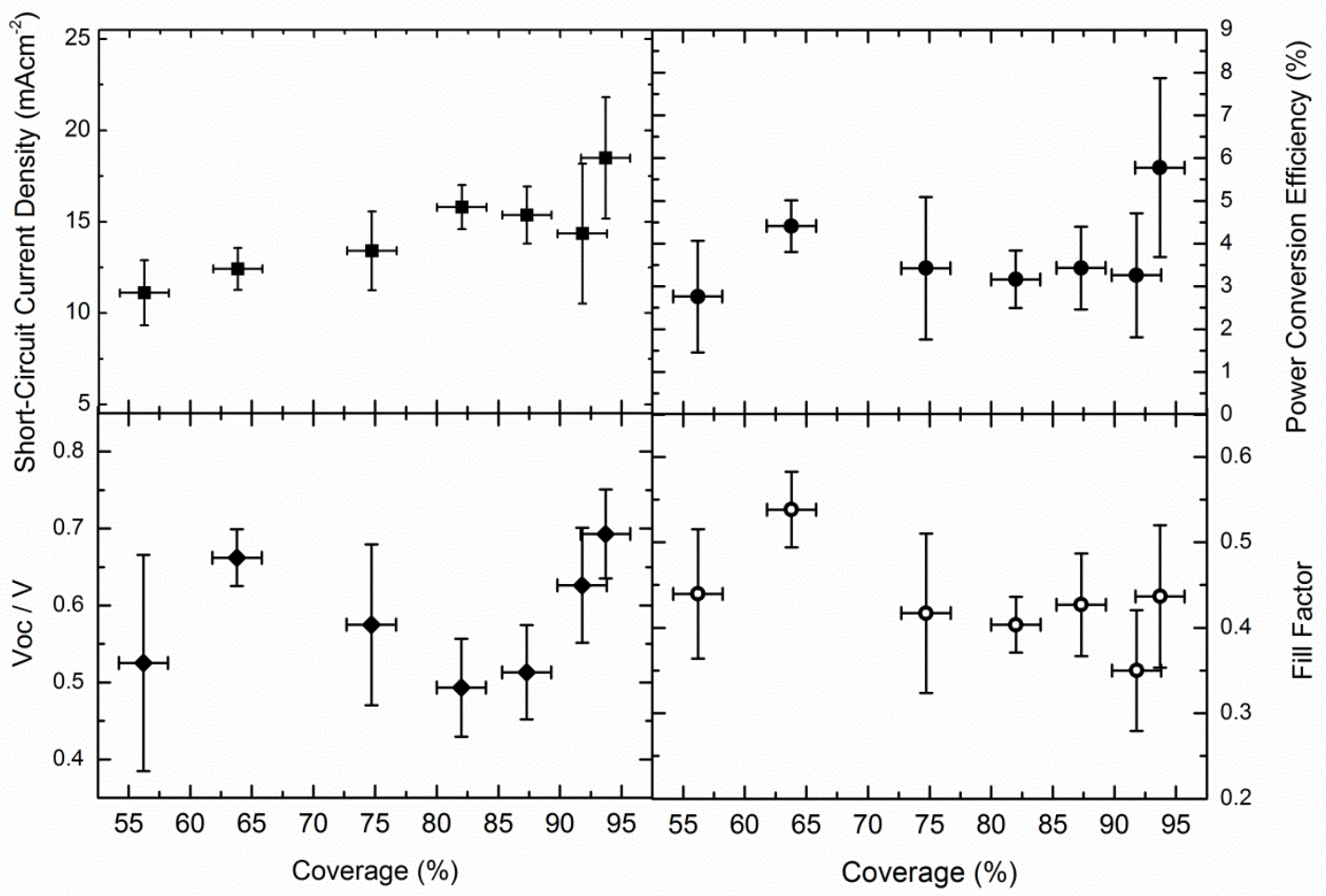

Figure S2. All device performance parameters extracted from current density-voltage data for the batch of solar cells with varying perovskite coverage described in the main text. 